\title{
ARTICLE
}

Received 9 Jun 2013 | Accepted 20 Aug 2013 | Published 17 Sep 2013

DOI: $10.1038 /$ ncomms3468

OPEN

\section{Spiral resonators for on-chip laser frequency stabilization}

\author{
Hansuek Lee ${ }^{1,2}$, Myoung-Gyun Suh', Tong Chen ${ }^{1}$, Jiang Li ${ }^{1}$, Scott A. Diddams ${ }^{3}$ \& Kerry J. Vahala ${ }^{1}$
}

Frequency references are indispensable to radio, microwave and time keeping systems, with far reaching applications in navigation, communication, remote sensing and basic science. Over the past decade, there has been an optical revolution in time keeping and microwave generation that promises to ultimately impact all of these areas. Indeed, the most precise clocks and lowest noise microwave signals are now based on a laser with short-term stability derived from a reference cavity. In spite of the tremendous progress, these systems remain essentially laboratory devices and there is interest in their miniaturization, even towards onchip systems. Here we describe a chip-based optical reference cavity that uses spatial averaging of thermorefractive noise to enhance resonator stability. Stabilized fibre lasers exhibit relative Allan deviation of $3.9 \times 10^{-13}$ at $400 \mu$ s averaging time and an effective linewidth $<100 \mathrm{~Hz}$ by achieving over $26 \mathrm{~dB}$ of phase-noise reduction.

\footnotetext{
${ }^{1}$ T.J. Watson Laboratory of Applied Physics, California Institute of Technology, Pasadena, California 91125, USA. ${ }^{2}$ hQphotonics, Pasadena, California 91106 , USA. ${ }^{3}$ Time and Frequency Division, National Institute of Standards and Technology, Boulder, Colorado 80305, USA. Correspondence and requests for materials should be addressed to K.J.V. (email: vahala@caltech.edu).
} 
pplications including gravity-wave detection ${ }^{1}$, optical clocks $^{2}$ and high-performance microwave generation ${ }^{3}$ have fuelled interest in frequency references for stabilization of laser sources. Such references benefit from high optical $Q$ factor or equivalently long optical storage time and systems in-use or under investigation include Fabry-Perot cavities $^{4-6}$, absorption spectral-hole burning in cryogenically cooled crystals ${ }^{7,8}$ and long-delay-line interferometers ${ }^{1,9}$. Benchtop-scale systems based on Fabry-Perot optical cavities have attained an Allan deviation $1 \times 10^{-16}$ at $1 \mathrm{~s}$ averaging ${ }^{5}$. In these systems, high-finesse-mirrors create a narrow resonance for laser locking, whereas low-thermal-expansion housings and lowthermal noise mirror coatings create immunity to thermal fluctuations that perturb the resonant frequency ${ }^{4-6,10,11}$. With the advent of ultra-high optical- $Q$, solid-state resonator systems based on silica ${ }^{12-14}$ and crystalline fluoride materials ${ }^{15-17}$ attention has naturally turned towards miniature devices. Besides their compact size, these devices, through their reduced mass, can offer improved performance with respect to shock and acceleration. In the case of chip-based devices, there is also the potential for integration with other components.

In solid-state resonators, fluctuations arise from thermorefractive, thermo-mechanical, elasto-optic and photo-thermal noise ${ }^{18-20}$. The first three mechanisms are fundamental, while the fourth is determined by the transfer of the laser power fluctuations into thermal changes of the cavity refractive index and size. Crystalline resonators are advantageous for reduced thermorefractive noise as the dependence of refractive index on temperature is low in comparison with silica ${ }^{19}$. Along these lines, locking of a laser to a $\mathrm{MgF}_{2}$ resonator has attained stabilization to $6 \times 10^{-14}$ at $0.1 \mathrm{~s}$ averaging time $\mathrm{e}^{21}$. It has also been demonstrated that dual-mode feedback control can be used to stabilize the absolute frequency of a resonator by measuring modal temperature using two, orthogonally polarized modes ${ }^{22-24}$. Moreover, application of these resonators as frequency stabilization elements in ring fibre lasers ${ }^{25,26}$ and designs for enhanced acceleration and vibration immunity have been proposed $^{27,28}$.

In this paper, we study the application of a chip-based, high- $Q$ resonator in the form of a spiral for laser frequency stabilization. Besides being the first chip-based reference cavities, the geometry offers a high level of immunity to thermorefractive noise, as well as thermo-mechanical and photo-thermal noise. Also, the measurements are performed without any special vacuum isolation and temperature control. Phase-noise spectra are measured and show strong laser phase-noise suppression over offset frequencies ranging from $1 \mathrm{~Hz}$ to $100 \mathrm{kHz}$. Frequency fluctuations, as characterized by the Allan deviation, are also measured.

\section{Results}

Design of low-thermal noise reference cavities. In the absence of resonator noise sources, the rms frequency difference of a locked laser relative to a cavity line center depends upon the optical $Q$ and signal-to-noise ratio (SNR) of the detected laser signal through the following expression ${ }^{29}$,

$$
\frac{\Delta v_{\mathrm{rms}}}{v_{0}} \approx \frac{\Delta v_{0}}{v_{0} \cdot \mathrm{SNR}}=\frac{1}{\mathrm{Q} \cdot \mathrm{SNR}}
$$

where $Q=v_{0} / \Delta v_{0}$ has been used in the result from the study by Drever et al. ${ }^{29}$ and where the SNR depends upon the integration time or servo-control bandwidth. Given a high enough $Q$ factor and large enough SNR, the stability of the laser locked to the cavity becomes determined by fluctuations in the cavity line centre, itself. Excluding technical noise sources such as acceleration and acoustics, the largest contribution to these fluctuations originates from thermorefractive noise $\mathrm{s}^{18,19}$. Intuitively, if one considers $\mathrm{N}$, randomly dispersed fluctuators that each contribute an rms frequency fluctuation $\sigma_{1}$, then the total $\mathrm{rms}$ frequency fluctuation will scale like $\sigma_{1} \sqrt{N}=\sigma_{1} \sqrt{\rho \cdot V}$ (assuming the fluctuators are uncorrelated) where $\rho$ is the density of fluctuators and $V$ is the mode volume. At the same time, if the fluctuators have a fixed size, then the coupling of the fluctuators to the mode will diminish as the mode volume increases and $\sigma_{1}$ will vary like $1 / V$. Therefore, the total $\mathrm{rms}$ frequency fluctuation will scale like $1 / \sqrt{V}$. This scaling is apparent in models of thermal-related fluctuations in resonators ${ }^{18,19}$. In optical fibre reference cavity systems, this source of noise is reduced by employing long fibre delays 9 .

To leverage this scaling on a silicon chip, a resonator in the form of a spiral is used (see Fig. 1). Spiral resonators have previously been used to create narrow free-spectral range devices on a chip ${ }^{30}$. To attain both high- $Q$ and large-mode volume a special ultra-low-loss waveguide is used in the current work, providing optical waveguide loss as low as $0.037 \mathrm{~dB} \mathrm{~m}^{-1}$ (refs 14,31). Using these waveguides we demonstrate resonators that are over $1 \mathrm{~m}$ in length with $Q$ factors in excess of 100 million, but for which the device footprint is smaller than $5.4 \mathrm{~cm}^{2}$. Beyond the thermorefractive noise immunity offered by these devices, they also provide enhanced immunity to photo-thermal noise. This happens because of their large mode volume, which greatly reduces the circulating intensity at a given coupled optical power and hence also the tendency for heating of the mode volume. As a result, it is possible to obtain high SNR (see equation. (1)) without degradation in resonator stability. Moreover, thermo-mechanical noise is greatly suppressed as this form of noise varies inversequadratically with resonator length ${ }^{32}$.

Representative long and short, round-trip path-length resonators are shown in Fig. 1. The resonators contain two interleaved spiral waveguides with $S$-turn adiabatic couplers at the spiral centre. Details on the process used to fabricate the waveguides are described in previous work ${ }^{31}$. Optical coupling to the resonators occurrs in the upper-right corner of the chip and uses a fibre taper ${ }^{33,34}$. Q measurements are performed by monitoring transmitted optical power on the taper coupler while scanning an external cavity semiconductor laser across a free-spectral range (FSR) of the resonator. The lower right inset of Fig. 2 shows a typical scan in the longest resonator having a round-trip physical path length of $120 \mathrm{~cm}$. The measured FSR of the device $(173 \mathrm{MHz})$ agrees well with the expected FSR based on the round-trip length. The other resonances seen in the inset

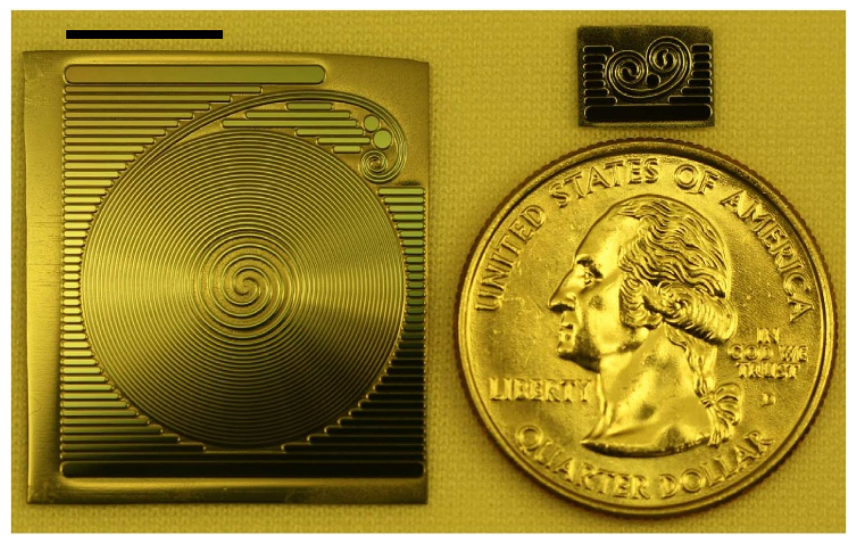

Figure 1 | Photograph of spiral waveguide resonators. Left: $1.2 \mathrm{~m}$ spiral resonator. Upper-right: $4.5 \mathrm{~cm}$ spiral resonator used for studies of $Q$ scaling. Lower right: quarter shown to provide scale. Scale bar, $1 \mathrm{~cm}$. 


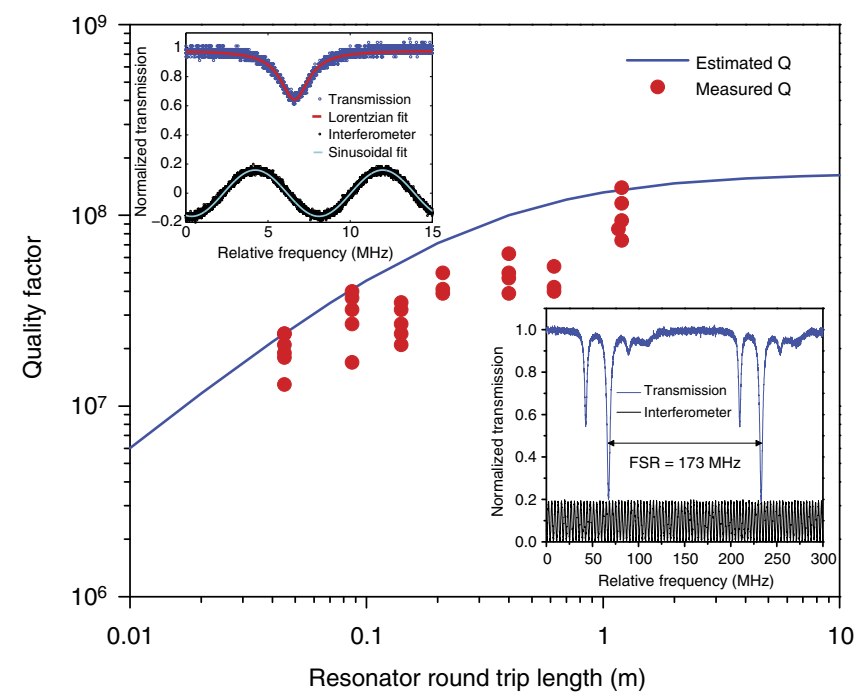

Figure 2 I Intrinsic $\mathbf{Q}$ factor measured for the various resonator lengths.

The maximum $Q$ factor obtained was 140 million at a length of $1.2 \mathrm{~m}$. The blue curve is a theoretical prediction for the $Q$ versus length that assumes a waveguide loss of $0.15 \mathrm{~dB} \mathrm{~m}^{-1}$. Upper left: a typical optical spectrum in blue. The sinusoidal curve is an interferometer scan that is used to calibrate the linewidth. Lower right: spectral scan in excess of one free-spectral range for the TE polarization. The black curve is the interferometer calibration trace.

correspond to higher-order transverse modes. The spectrum is remarkably uncluttered. We attribute this to spatial filtering of higher-order transverse modes by the adiabatic couplers ( $S$-bend waveguide turns at the centre of each spiral) ${ }^{35}$. To verify the dependence of $Q$ factor on resonator length a range of device lengths are tested $(4.5,8.7,14,21,40,62$ and $120 \mathrm{~cm})$. The results are plotted in the main panel of Fig. 2 along with a theoretical estimate of the $Q$ factor based upon an adiabatic coupler loss of $0.02 \mathrm{~dB}$ per coupler and a waveguide loss of $0.15 \mathrm{~dB} \mathrm{~m}^{-1}$. The agreement is reasonable. Also, the waveguide loss here is higher than reported in earlier work on account of using a contact aligner for micro-fabrication as opposed to a projection (stepper) lithography system ${ }^{14,31}$. Nonetheless, a maximum $Q$ factor of 140 million is obtained.

Phase-noise spectrum and linewidth. To measure the frequency stability of the spiral resonators, the experimental setup shown in Fig. 3 was used. It includes two, fibre lasers (Orbits Lightwave, at optical frequencies near $193.43 \mathrm{THz}$ ) that are locked with separate Pound-Drever-Hall systems ${ }^{29}$ to two, high-Q, silica-on-silicon spiral resonators. In the measurements, $\sim 3 \mathrm{~mW}$ of laser power is in the fibre-taper waveguide and about $1 \mathrm{~mW}$ of this power is coupled into the resonator. The locked fibre lasers were heterodyned to produce a beat signal near $350 \mathrm{MHz}$. This beat note directly reveals the combined phase-noise of the two stabilized lasers, and it was analysed using an electrical spectrum analyser, a phase-noise analyser (Rohde \& Schwarz FSUP26) and a frequency counter (Tektronix FCA3120 and Pendulum CNT91). Acoustic shielding was placed around the entire setup to attenuate environmental sound; also, pumps and instrumentation in adjoining rooms were turned-off during measurements. Measurements were performed for several cases: free-running fibre lasers, lasers locked to the $1.2 \mathrm{~m}$ spiral reference cavities, and, for comparison purposes, lasers locked to independent conventional disk resonators of varying diameters (3, 7.5 and $15 \mathrm{~mm})^{14}$. In prior work, linear drift has been substracted

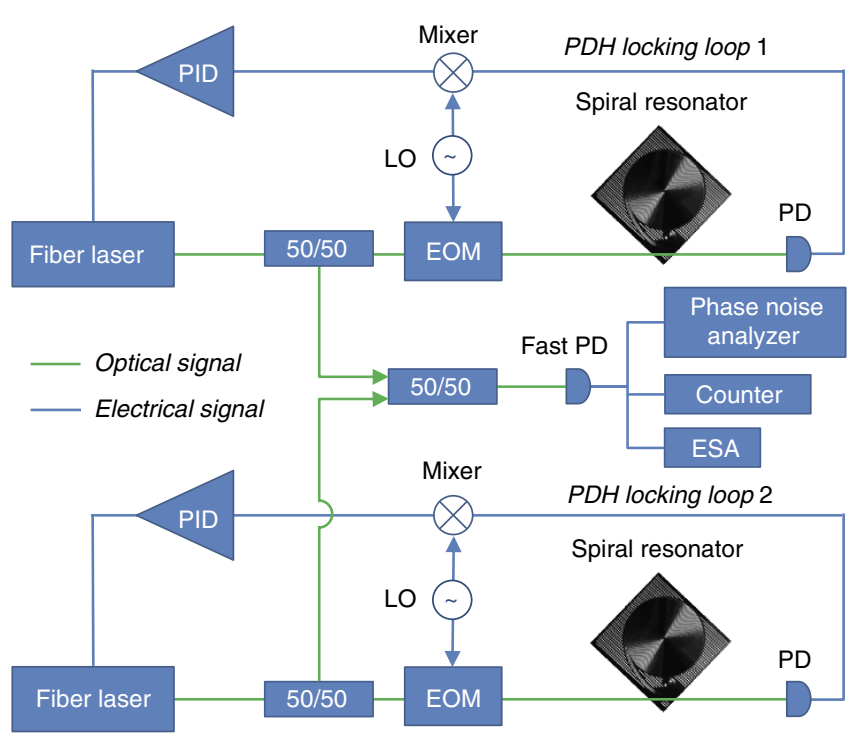

Figure 3 | Experimental setup. Two, fibre lasers independently locked to high-Q spiral reference cavities using Pound-Drever-Hall (PDH) locking systems. Each PDH locking loop includes a photodiode (PD), electro-optic modulator (EOM), local oscillator (LO) and proportional-integral-

differential feedback controller (PID). The outputs of the separately locked lasers were combined on a photodetector and the resulting photocurrent was analysed using an electrical spectrum analyser (ESA), a frequency counter and a phase-noise analyser. All components are on the same optical table.

from data ${ }^{9,21}$. In the present work, no linear drift correction was performed.

Figure 4a shows the phase-noise spectral density function for the heterodyned signals both with and without the locking systems engaged. Data are shown for the free-running lasers, $3 \mathrm{~mm}$ disk resonators and the $1.2 \mathrm{~m}$ spiral cases. The spectra were measured over offset frequencies from $1 \mathrm{~Hz}$ to $10 \mathrm{MHz}$ and an instrument smoothing algorithm has been applied to show the trend. Within the bandwidth of the feedback control system (bandwidth limit is delineated by the servo-control bumps visible near $200 \mathrm{kHz}$ in the phase-noise spectrum) an average of $26 \mathrm{~dB}$ suppression of fibre-laser phase-noise was measured when the fibre lasers were locked to the $1.2 \mathrm{~m}$ spirals. In comparison, only $10 \mathrm{~dB}$ of suppression was achieved with the $3 \mathrm{~mm}$ diameter disks (measured at $1 \mathrm{kHz}$ offset frequency). Below $1 \mathrm{kHz}$ offset frequency even better suppression was observed for spiral locking versus disk locking. We believe this is caused by better immunity to photo-thermal noise in the spiral resonators on account of their larger mode volume. For example, the $3 \mathrm{~mm}$ disk, phasenoise spectrum degrades at offset frequencies less than $1 \mathrm{kHz}$, which is consistent with the thermal corner frequency observed in other silica-based resonators ${ }^{36}$. In the inset of Fig. $4 \mathrm{a}$, the noise suppression improvement relative to the free-running fibre laser case is plotted for each of the resonators measured. The data here are taken at $1 \mathrm{kHz}$ offset and also at $100 \mathrm{~Hz}$ offset to illustrate the improved suppression of noise at lower offset frequency provided by the spiral resonator. Overall, there is roughly a $1 / f^{3}$ dependence of phase-noise on frequency. This is indicative of flicker noise and the dependence is consistent with resonator modelling of thermorefractive noise, which generally feature a roll-off in frequency that is faster than $1 / f^{2}$ down to low-offset frequencies $^{18,19}$.

Figure $4 \mathrm{~b}$ shows a comparison of the measured electrical spectrum generated upon heterodyne detection with the 

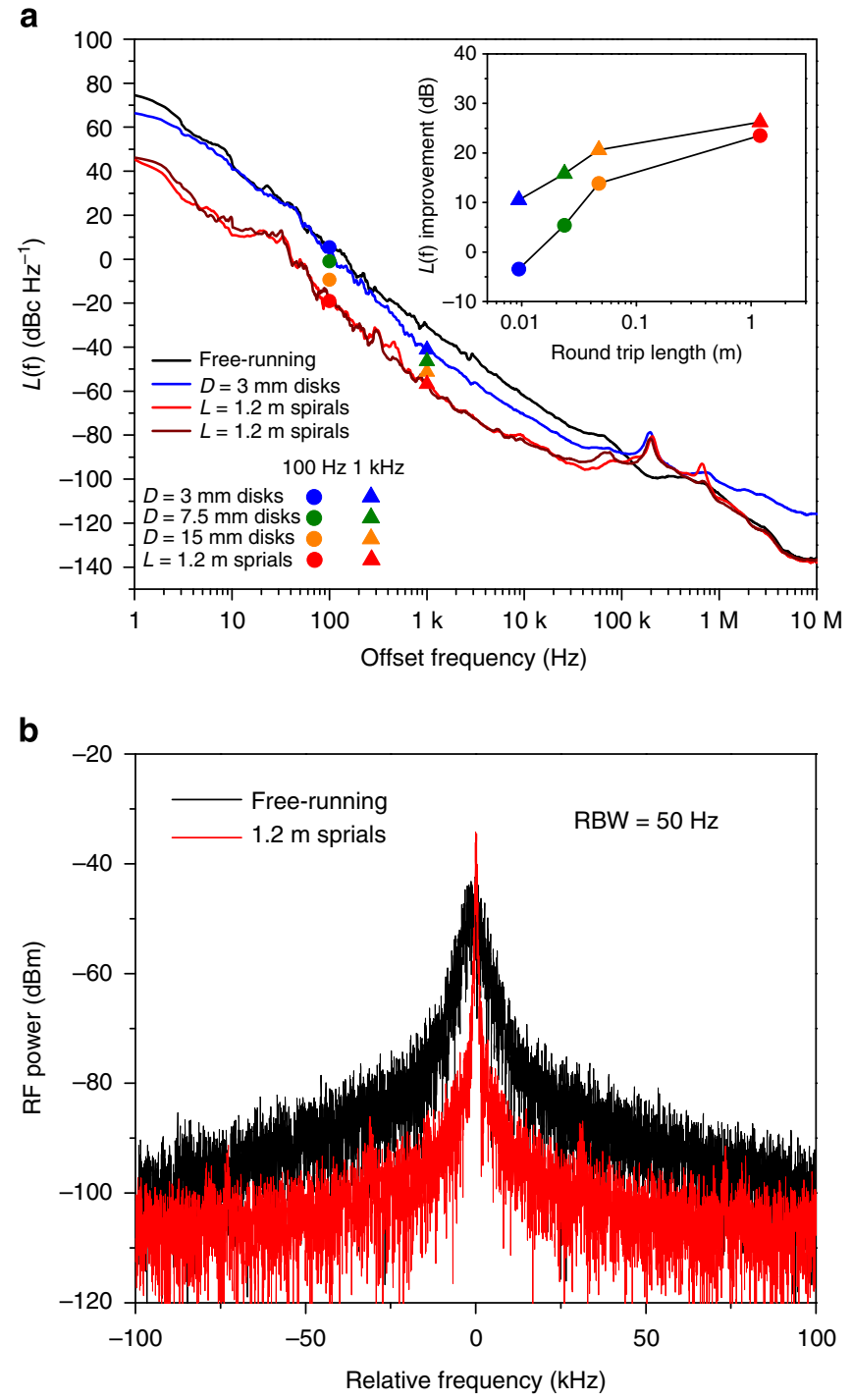

Figure 4 | Phase-noise spectra and beat-note spectral measurement. (a) Phase-noise spectra measured for two, free-running $193 \mathrm{THz}$ fibre lasers (black), fibre lasers independently locked to two, $3 \mathrm{~mm}$ disk resonators (blue), and fibre lasers independently locked to two, $1.2 \mathrm{~m}$ long spiral resonators (red). To test measurement reproducibility, resonators were characterized on multiple days. Measurement of the $1.2 \mathrm{~m}$ long resonators on a second day is shown as the dark-red trace. The data for the spiral resonator show an average suppression by $26 \mathrm{~dB}$ of the fibre laser noise when locked to the spiral resonators. In comparison, $10 \mathrm{~dB}$ of noise suppression is observed using the $3 \mathrm{~mm}$ device. The servo-control noise bumps appear at around $200 \mathrm{kHz}$ for the locked phase-noise spectra. The inset is a plot of the noise suppression at $100 \mathrm{~Hz}$ and $1 \mathrm{kHz}$ offset frequencies plotted versus resonator length for each of the resonators tested. (b) The electrical spectrum of the fibre lasers' beat note for both free-running and locked configurations. Spectral narrowing and noise suppression are apparent in the locked spectrum.

free-running fibre lasers to the case when the lasers are independently locked to the $1.2 \mathrm{~m}$ long resonators. For this measurement, the resolution bandwidth (RBW) of the electrical spectrum analyser was set to $50 \mathrm{~Hz}$, resulting in an $80 \mathrm{~ms}$ sweep time over the $200 \mathrm{kHz}$ span. As an additional comparison, we have calculated the effective linewidths for the beat note of the two, stabilized lasers from the phase-noise spectra ${ }^{37}$ and found $900 \mathrm{~Hz}$ (free-running lasers), $400 \mathrm{~Hz}$ (locked to $3 \mathrm{~mm}$ disks) and

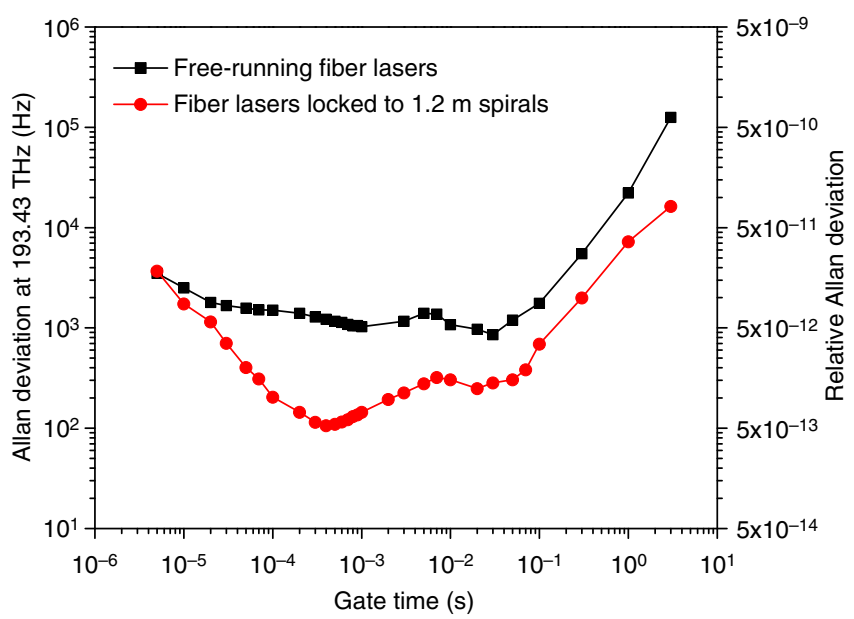

Figure 5 | Allan deviation measurement result. Allan deviation of the beat frequency between the two, free-running fibre lasers (black squares), and for the lasers independently locked to two, $1.2 \mathrm{~m}$ long spiral resonators (red circles) is shown. A minimum Allan deviation of $100 \mathrm{~Hz}$ at an optical frequency of $193 \mathrm{THz}$, corresponding to a relative Allan deviation of $5.5 \times 10^{-13}$, was measured at a gate time of $400 \mu \mathrm{s}$ for $10 \mathrm{~dB}$ improvement compared with the free-running case. Assuming the fibre lasers are independent and equivalent, a relative Allan deviation of $3.9 \times 10^{-13}$ is expected for each locked laser.

$100 \mathrm{~Hz}$ (locked to $1.2 \mathrm{~m}$ spiral resonators). The individual laser linewidths will be narrower than these values. The calculated, beatnote linewidth of the lasers locked to $1.2 \mathrm{~m}$ spiral resonators is consistent with both the electrical spectrum measurement of the beatnote as well as the Allan deviation measurement result.

Allan deviation measurement. In order to further confirm the frequency stabilization by the spiral resonators, Allan deviation measurements ${ }^{38}$ were carried out using a Tektronix FCA3120 frequency counter (Fig. 5). As an additional check the measurements were also confirmed using a Pendulum CNT-91 frequency counter. Zero dead-time measurements were performed using both frequency counters. Over the range of gate times from $5 \mu \mathrm{s}$ to $3 \mathrm{~s}$, Allan deviations of the spiral-locking case were improved in comparison with the free-running (unlocked) case. At a gate time of $400 \mu \mathrm{s}$, a minimum relative Allan deviation of $5.5 \times 10^{-13}$ was measured, which is ten times lower than that of the free-running case. If the two lasers are assumed to be independent, then the relative deviation of a single, stabilized laser is $3.9 \times 10^{-13}$, or an equivalent Allan deviation of $75 \mathrm{~Hz}$. After this minimum there is a rise, then decrease and final rise in the Allan deviation. The resulting local maximum near $10 \mathrm{~ms}$ is believed to result from environmental fluctuations and to also be associated with the corresponding increase around $10-60 \mathrm{~Hz}$ in the phasenoise spectrum in Fig. 4a. It was also noteworthy that stability improvement of locked signal at longer gate times is consistent with the phase-noise suppression at low-offset frequency $(<10 \mathrm{~Hz})$.

Mechanically induced noise. Measurements of thermomechanical-induced noise were also conducted using both the disk and spiral resonators. The optomechanical coupling parameter is expected to vary inversely with cavity length so that phase-noise exhibits an inverse quadratic dependence on length $^{32}$. This dependence was observed over a range of cavity lengths by using the Hänsch Couillard technique ${ }^{39,40}$. Spectral 
features believed to be thermally excited mechanical resonances were observed at offset frequencies greater than $1 \mathrm{MHz}$, and steadily diminished in amplitude to levels below the sensitivity limit of the system for the largest spirals measured $(1.2 \mathrm{~m}$ path length). As confirmed in Fig. 4a, there was no evidence of mechanical noise in the phase-noise spectra measurements.

\section{Discussion}

It is noteworthy that ideal frequency division of the $193 \mathrm{THz}$ optical carrier to $10 \mathrm{MHz}$ would provide a signal with close-tocarrier phase-noise of $\sim-100 / f^{3} \mathrm{dBc} \mathrm{Hz}^{-1}$. This is a level that is already competitive with the state-of-the-art oven-controlled crystal oscillators, and the basic architecture to accomplish this has been demonstrated with the combination of laboratory frequency combs and electronic division ${ }^{41}$. It is intriguing to consider that the spiral cavity demonstrated here could be the frequency reference for a chip-integrated platform, that together with advances in microcomb technology ${ }^{42,43}$ would ultimately provide broad-bandwidth synthesis of low-phase-noise signals from the optical to the RF. In addition, the ability to reduce the effective linewidth of a fibre laser by a factor of $10 \mathrm{X}$ using only a chip-based device is of practical importance in any applications requiring high coherence. This includes coherent fibre-optic communications $^{44,45}$, remote sensing ${ }^{46}$ and atomic physics ${ }^{4,47}$. Moreover, aside from simple acoustical shielding of the experimental setup and operation on a floated optical table, there has been no attempt to thermally stabilize or vibration isolate these devices. Likewise, there has been no drift correction of the data. Concerning future performance improvements, optical-fibre-based reference systems using $1 \mathrm{~km}$ fibre delays have attained a phase-noise level of $-83 \mathrm{dBc} \mathrm{Hz}^{-1}$ at $1 \mathrm{kHz}$ offset frequency 9 . In the current chip-based design, 27 metre long delay lines have been demonstrated and lengths in excess of $100 \mathrm{~m}$ are feasible ${ }^{31}$. Finally, thicker oxides may be possible if thermal oxidation is replaced by processes such as the flame hydrolysis method. The combination of these methods could produce a 1,000 -fold increase in mode volume relative to the current results.

\section{References}

1. Abbott, B. P. et al. LIGO: the laser interferometer gravitational-wave observatory. Rep. Prog. Phys. 72, 076901 (2009).

2. Diddams, S. A. et al. An optical clock based on a single trapped ${ }^{199} \mathrm{Hg}^{+}$ion. Science 293, 825-828 (2001).

3. Fortier, T. M. et al. Generation of ultrastable microwaves via optical frequency division. Nat. Photon. 5, 425-429 (2011).

4. Young, B. C., Cruz, F. C., Itano, W. M. \& Bergquist, J. C. Visible lasers with subhertz linewidths. Phys. Rev. Lett. 82, 3799-3802 (1999).

5. Kessler, T. et al. A sub-40-mHz-linewidth laser based on a silicon single-crystal optical cavity. Nat. Photon. 6, 687-692 (2012).

6. Jiang, Y. Y. et al. Making optical atomic clocks more stable with $10^{-16}$-level laser stabilization. Nat. Photon. 5, 158-161 (2011).

7. Sellin, P. B., Strickland, N. M., Carlsten, J. L. \& Cone, R. L. Programmable frequency reference for subkilohertz laser stabilization by use of persistent spectral hole burning. Opt. Lett. 24, 1038-1040 (1999).

8. Thorpe, M. J., Rippe, L., Fortier, T. M., Kirchner, M. S. \& Rosenband, T. Frequency stabilization to $6 \times 10^{-16}$ via spectral-hole burning. Nat. Photon. 8, 688-693 (2011).

9. Kefelian, F., Jiang, H., Lemonde, P. \& Santarelli, G. Ultralow-frequency-noise stabilization of a laser by locking to an optical fibre-delay line. Opt. Lett. 34, 914-916 (2009).

10. Numata, K., Kemery, A. \& Camp, J. Thermal-noise limit in the frequency stabilization of lasers with rigid cavities. Phys. Rev. Lett. 93, 250602 (2004).

11. Harry, G. M. et al. Thermal noise in interferometric gravitational wave detectors due to dielectric optical coatings. Class. Quant. Grav. 19, 897-917 (2002).

12. Armani, D. K., Kippenberg, T. J., Spillane, S. M. \& Vahala, K. J. Ultra-high-Q toroid microcavity on a chip. Nature 421, 925-928 (2003).

13. Papp, S. B. \& Diddams, S. A. Spectral and temporal characterization of a fusedquartz-microresonator optical frequency comb. Phys. Rev. A 84, 053833 (2011).
14. Lee, H. et al. Chemically etched, ulta-high-Q resonator on a chip. Nat. Photon. 6, 369-373 (2012).

15. Grudinin, I. S., Ilchenko, V. S. \& Maleki, L. Ultrahigh optical Q factors of crystalline resonators in the linear regime. Phys. Rev. A 74, 063806 (2006).

16. Grudinin, I. S., Matsko, A. B. \& Maleki, L. On the fundamental limits of Q factor of crystalline dielectric resonators. Opt. Exp. 15, 3390-3395 (2007).

17. Savchenkov, A. A., Matsko, A. B., Ilchenko, V. S. \& Maleki, L. Optical resonators with ten million finesse. Opt. Exp. 15, 6768-6773 (2007).

18. Gorodetsky, M. L. \& Grudinin, I. S. Fundamental thermal fluctuations in microspheres. J. Opt. Soc. Am. B 21, 697-705 (2004).

19. Matsko, A. B., Savchenkov, A. A., Yu, N. \& Maleki, L. Whispering-gallery-mode resonators as frequency references. I. fundamental limitations. J. Opt. Soc. Am. B 24, 1324-1335 (2007).

20. Chijioke, A., Chen, Q., Nevsky, A. Y. \& Schiller, S. Thermal noise of whispering-gallery resonators. Phys. Rev. A 85, 053814 (2012).

21. Alnis, J. et al. Thermal-noise-limited crystalline whispering-gallery-mode resonator for laser stabilization. Phys. Rev. A 84, 011804 (2011).

22. Savchenkov, A. A., Matsko, A. B., Ilchenko, V. S., Yu, N. \& Maleki, L. Whispering-gallery-mode resonators as frequency references. II. stabilization. J. Opt. Soc. Am. B 24, 2988-2997 (2007)

23. Strekalov, D. V., Thompson, R. J., Baumgartel, L. M., Grudinin, I. S. \& Yu, N Temperature measurement and stabilization in a birefringent whispering gallery mode resonator. Opt. Exp. 19, 14495-14501 (2011).

24. Fescenko, I. et al. Dual-mode temperature compensation technique for laser stabilization to a crystalline whispering gallery mode resonator. Opt. Exp. 20, 19185-19193 (2012).

25. Sprenger, B., Schwefel, H. G. L., Lu, Z. H., Svitlov, S. \& Wang, L. J. CaF 2 whispering-gallery-mode-resonator stabilized-narrow-linewidth laser. Opt. Lett. 35, 2870-2872 (2010).

26. Collodo, M. C. et al. Sub-khz lasing of a $\mathrm{CaF}_{2}$ whispering gallery mode resonator stabilized fibre ring laser. Preprint at http://arxiv.org/abs/1208.0245 (2012).

27. Chembo, Y. K., Baumgartel, L. M. \& Yu, N. Toward whispering-gallery-mode disk resonators for metrological applications. In SPIE News Sensing and Measurement (2012), doi:10.1117/2.1201202.004034.

28. Chembo, Y. K., Baumgartel, L. M. \& Yu, N. Exploring the frequency stability limits of whispering gallery mode resonators for metrological applications. in Proc. SPIE 8236, Laser Resonators, Microresonators, and Beam Control XV, 82360Q, doi:10.1117/12.908990 (2012).

29. Drever, R. W. P. et al. Laser phase and frequency stabilization using an optical resonator. Appl. Phys. B 31, 97-105 (1983).

30. Xu, D.-X. et al. Archimedean spiral cavity ring resonators in silicon as ultracompact optical comb filters. Opt. Exp. 18, 1937-1945 (2010).

31. Lee, H., Chen, T., Li, J., Painter, O. \& Vahala, K. Ultra-low-loss optical delay line on a silicon chip. Nat. Commun. 3, 867 (2012).

32. Kippenberg, T. J. \& Vahala, K. J. Cavity optomechanics: back-action at the mesoscale. Science 321, 1172-1176 (2008).

33. Cai, M., Painter, O. \& Vahala, K. J. Observation of critical coupling in a fibre taper to silica-microsphere whispering gallery mode system. Phys. Rev. Lett. 85, 74-77 (2000)

34. Spillane, S. M., Kippenberg, T. J., Painter, O. J. \& Vahala, K. J. Observation of critical coupling in a fibre taper to silica-microsphere whispering gallery mode system. Phys. Rev. Lett. 85, 74-77 (2000)

35. Chen, T., Lee, H., Li, J. \& Vahala, K. A general design algorithm for low optical loss adiabatic connections in waveguides. Opt. Exp. 20, 22819-22829 (2012).

36. Rokhsari, H. \& Vahala, K. J. Observation of kerr nonlinearity in microcavities at room temperature. Opt. Lett. 427-429 (2005).

37. Hjelme, D. R., Mickelson, A. R. \& Beausoleil, R. G. Semiconductor laser stabilization by external optical feedback. J. Qunt. Electron. 27, 352-372 (1991).

38. Barnes, J. A. et al. Characterization of frequency stability. IEEE Trans. Instrum. Meas. 20, 105-120 (1971).

39. Hänsch, T. W. \& Couillaud, B. Laser frequency stabilization by polarization spectroscopy of a reflecting reference cavity. Opt. Commun. 35, 441-444 (1980).

40. Schliesser, A., Riviere, R., Anetsberger, G., Arcizet, O. \& Kippenberg, T. J Resolved-sideband cooling of a micromechanical oscillator. Nat. Phys. 4, 415-419 (2008).

41. Hati, A. et al. Ultra-low-noise regenerative frequency divider for high-spectralpurity RF signal generation. In Frequency Control Symposium (FCS), 2012 IEEE International (2012).

42. Del'Haye, P. et al. Optical frequency comb generation from a monolithic microresonator. Nature 450, 1214-1217 (2007).

43. Kippenberg, T. J., Holzwarth, R. \& Diddams, S. A. Microresonator-based optical frequency combs. Science 332, 555-559 (2011).

44. Ip, E., Lau, A., Barros, D. \& Kahn, J. Coherent detection in optical fibre systems. Opt. Exp. 16, 753-791 (2008) 
45. Koizumi, Y. et al. 256-QAM (64 Gb/s) coherent optical transmission over $160 \mathrm{~km}$ with an optical bandwidth of $5.4 \mathrm{GHz}$. Photon. Technol. Lett. 22, 185-187 (2010).

46. Karlsson, C. J., Olsson, F. A. A., Letalick, D. \& Harris, M. All-fibre multifunction continuous-wave coherent laser radar at $1.55 \mu \mathrm{m}$ for range, speed, vibration, and wind measurements. Appl. Opt. 39, 3716-3726 (2000).

47. Rafac, R. J. et al. Sub-dekahertz ultraviolet spectroscopy of ${ }^{199} \mathrm{Hg}^{+}$. Phys. Rev. Lett. 85, 2462-2465 (2000).

\section{Acknowledgements}

We thank Andrew Ludlow and Scott Papp (NIST, Boulder CO) for helpful discussions and comments on this manuscript. We gratefully acknowledge the Defence Advanced Research Projects Agency under SB121-001, the iPhoD program, and also the QuASAR program, the Kavli Nanoscience Institute and the Institute for Quantum Information and Matter, an NSF Physics Frontiers Centre with support of the Gordon and Betty Moore Foundation. The views expressed are those of the authors and do not reflect the official policy or position of the Department of Defence or the US Government. Distribution A-approved for public release; distribution is unlimited.

\section{Author contributions}

H.L., T.C. and K.J.V. conceived the devices and all authors helped to design the experiment. H.L. fabricated the devices with assistance from T.C. M.G.S. measured the devices with assistance from the other authors. All authors helped to write the paper.

\section{Additional information}

Competing financial interests Two authors declare competing financial interests. H.L. is an employee of hQphotonics. H.L. and K.V. are founders of hQphotonics.

Reprints and permission information is available online at http://npg.nature.com/ reprintsandpermissions/

How to cite this article: Lee, H. et al. Spiral resonators for on-chip laser frequency stabilization. Nat. Commun. 4:2468 doi: 10.1038/ncomms3468 (2013).

(c) This article is licensed under a Creative Commons Attribution 3.0
Unported Licence. To view a copy of this licence visit http:// creativecommons.org/licenses/by/3.0/. 